\title{
AMBIENTES PROTEGIDOS PARA EL ALMACENAMIENTO TEMPORAL Y EL SECADO DEL FRIJOL (Phaseolus vulgaris L.) EN EL CAMPO ${ }^{1}$
}

\author{
Marco Vinicio Gutiérrez-Soto², Néstor Chaves-Barrantes ${ }^{2}$, Juan Carlos Hernández-Fonseca ${ }^{3}$, \\ Rodolfo Araya-Villalobos ${ }^{2}$, Diego Ureña-Solís ${ }^{2}$
}

\section{RESUMEN}

Ambientes protegidos para el almacenamiento temporal y el secado del frijol (Phaseolus vulgaris L.) en el campo. EEl objetivo de este trabajo fue evaluar ambientes protegidos bioclimáticos para el almacenamiento temporal y el secado de frijol en el campo, en Pérez Zeledón y Buenos Aires, Costa Rica, durante la estación lluviosa del año 2007. El diseño propuesto es una modificación de las estructuras rústicas empleadas por los agricultores, y utiliza la ventilación cruzada y materiales reflectivos, mediante la elevación del piso donde se coloca el frijol y la eliminación de paredes laterales. Se evaluaron tres colores de coberturas plásticas: transparente, negro y gris, de $0,0038 \mathrm{~cm}$ de grosor, y se empleó como tratamiento testigo el diseño con plástico transparente usado por los agricultores (cuatro tratamientos). Se midió la temperatura y la humedad en el interior del material vegetal colocado dentro de las estructuras $(0,20 \mathrm{y}$ $1,25 \mathrm{~m}$ de profundidad) y en el ambiente exterior durante 16 días, con dos periodos de mediciones de ocho días consecutivos cada uno y tres repeticiones (tres fincas). Se evaluó además el contenido de humedad y el tiempo de cocción de los granos. Los materiales plásticos reflectivos y el diseño bioclimático mejoraron el microclima en el interior de las estructuras de almacenamiento y se redujo significativamente el tiempo de cocción de los frijoles, en comparación con los diseño tradicional con plástico trasparente empleado por los agricultores.

Palabras clave: Calidad de los granos, coberturas sintéticas, diseño bioclimático, fitomejoramiento participativo, tiempo de cocción.

\begin{abstract}
Shelters for the temporal storage and drying of common beans (Phaseolus vulgaris L.) in the field. The objective of this work was to evaluate sheltered environments for temporal storage and drying in the field of common beans harvested in hill-side agriculture in Mesoamerica. This study was conducted in Pérez Zeledón and Buenos Aires during the rainy season of year 2007. The proposed design modifies the rustic structures traditionally used by farmers, includes cross-ventilation, floor elevation, remotion of lateral walls, and low-cost, locally-available materials. We also applied principles of energy balance and used reflective materials that reduced energy and heat load. Three colors of plastic covers were evaluated: transparent, black and gray, of $0,0038 \mathrm{~cm}$ thickness. The control treatment was the design and plastic cover used by farmers (four treatments). We measured temperature and relative humidity inside $(0,20$ and 1,25 m deepth) and outside the shelters during 16 days, over two measurement periods of 8 consecutive days each, in three farms (replications). Seed humidity and cooking time were also evaluated. Reflective plastic materials and the bioclimatic design deployed significantly improved the microclimate within the shelters, and resulted in substantial reductions in cooking time of bean seeds in comparison with the traditional design with transparent plastic used by farmers.
\end{abstract}

Key words: Seed quality, synthetic cover materials, bioclimatic design, participative plant breeding, cooking time.

\footnotetext{
1 Recibido: 8 de diciembre, 2008. Aceptado: 16 de noviembre, 2009.

2 Universidad de Costa Rica, Estación Experimental Fabio Baudrit, Apdo. 183-4050 Alajuela, Costa Rica. Teléfonos: (506) 2433-8284, 25117798, Fax: (506) 2433 9086. surdo26@racsa.co.cr; nfchaves@gmail.com; 1die2001@yahoo.com; avillalo2005@hotmail.com

3 Instituto Nacional de Innovación y Transferencia Tecnológica Agropecuaria (INTA). San José, Costa Rica. j.hernandez@ costarricense.cr
} 


\section{INTRODUCCIÓN}

El frijol común (Phaseolus vulgaris L.) es la leguminosa alimenticia más importante para el consumo humano directo a nivel mundial, y es cultivado mayormente en minifundios con pocos insumos, bajo un amplio rango de sistemas de producción y ambientes. En los trópicos americanos, el cultivo del frijol está sujeto a numerosos factores limitantes de naturaleza biológica, edáfica y climática (Pastor-Corrales y Schwartz 1994). El frijol se cultiva mayormente bajo la modalidad de agricultura dependiente de la lluvia, frecuentemente en terrenos poco fértiles, con pendientes pronunciadas, donde la degradación ambiental debida a la erosión hídrica y eólica es más severa (Bertsch y Monreal 1996).

Los granos de frijol cosechados, son además propensos al endurecimiento, particularmente si se reduce su humedad rápidamente y en condiciones de altas temperaturas, lo que provocan aumentos del tiempo de cocción y reducen la calidad y el precio de los granos (Mora 1982 y 1989). Breves períodos bajo condiciones inadecuadas de almacenamiento pueden conducir al rápido deterioro de los granos del frijol y otras leguminosas, antes de que éstos alcancen las redes de conservación y distribución de alimentos (Mora 1982, Reyes-Moreno et al. 2000). La domesticación de $P$. vulgaris ha incluido la pérdida de la latencia de los granos y el consecuente viviparismo (Bewley y Black 1994), y aunque la eliminación de la latencia permite la germinación sincronizada y la reducción del tiempo de cocción, también puede resultar en la germinación precoz dentro de las vainas si los granos están cerca de su madurez fisiológica, o durante el almacenamiento, si las condiciones de humedad permiten la imbibición.

Dadas las limitaciones financieras y ambientales involucradas en la producción de granos básicos como el frijol, es necesario implementar soluciones tecnológicas simples y sostenibles, que garanticen la conservación de la calidad post-cosecha de los productos luego de un costoso y largo proceso de producción en el campo. En el caso del frijol común, el almacenamiento temporal en el campo y el secado inicial de los granos son realizados por los agricultores con estructuras rústicas (PRIAG 1996) que causan alta temperatura, rápidas reducciones de la humedad de los granos, alto tiempo de cocción, y pérdidas de la calidad, la viabilidad, y el valor nutricional de los mismos. En esta especie, se recomienda la cosecha cuando el contenido de agua de los granos está entre $18-20 \%$, su trillado entre $14-15 \%$, y su almacenamiento con $12 \%$ de humedad. Sin embargo, si los granos se calientan excesivamente durante el secado, los riesgos de endurecimiento aumentan considerablemente (CIAT 1980). Mora (1982) ha advertido que la relación entre el contenido de agua y el tiempo de cocción de los frijoles no es única, sino que es afectada por varios factores como la velocidad y la temperatura de secado, y Sánchez-Trejos (2006) ha señalado también los efectos del tipo y duración del almacenamiento sobre la calidad nutricional del frijol.

La construcción y el funcionamiento de secadores solares o de leña, carbón u otros combustibles fósiles recomendados para el secado de los frijoles (Mora 1981) implican costos energéticos, financieros y ambientales que los hacen insostenibles en la agricultura de subsistencia y en otros agro-ecosistemas que operan con pocos insumos externos, en sitios donde la degradación ambiental ha causado la pérdida de los recursos renovables locales. En contraposición, las estructuras tradicionalmente empleadas por los agricultores para la protección pasiva y el secado controlado de los granos en el campo, demuestran principios fundamentales del diseño bioclimático (Gwinner et al. 1996, van Lengen 2004), e incluyen la reducción de la carga energética, el uso de materiales aislantes, y la ventilación cruzada.

En este trabajo se evaluó el desempeño de un diseño pasivo que utiliza el clima natural para el secado y la protección temporal de frijoles en el campo. Se implementó un ambiente protegido diseñado con base en principios físicos simples y consideraciones bioclimáticas (van Lengen 2004, Stagno y Gutiérrez-Soto 2005), que además involucró a los agricultores en el proceso de diseño e implementación en sus propias fincas. El diseño mejorado utiliza el clima natural para promover la ventilación y el secado, protege la cosecha de la lluvia y el rocío, y reduce el endurecimiento de los frijoles y las pérdidas post-cosecha. El ambiente protegido modificado introduce un piso permeable elevado y coberturas reflectivas que reducen la carga energética y amortiguan la temperatura. Es además ambientalmente sostenible, duradero, multi-uso, semipermanente, fácil de ensamblar, y compuesto en su mayoría de materiales naturales y sintéticos reutilizables y de bajo costo. 


\section{MATERIALES Y MÉTODOS}

\section{Sitio del estudio y condiciones agroclimáticas}

La investigación se llevó a cabo durante la estación lluviosa del año 2007, en fincas de productores ubicadas en Veracruz ( $9^{\circ} 06^{\prime} \mathrm{N}$; $83^{\circ} 32^{\prime} \mathrm{O}, 539 \mathrm{~m}$ ), Las Delicias de Pejibaye, Pérez Zeledón (09 06' N; 83 31' O; $662 \mathrm{~m})$, y Concepción de Pilas, Buenos Aires $\left(09^{\circ}\right.$ $05^{\prime} \mathrm{N}$; 83 29'; 706 m), Costa Rica. Estas comunidades se destacan por su importancia en la producción de frijol, maíz y ganado en la región Brunca de Costa Rica. Cerca del 100\% de los productores cultivan granos básicos en sus sistemas de producción, en fincas menores a 5 ha y ubicadas en terrenos con pendientes pronunciadas. En la zona habitan 400 agricultores y sus familias, que siembran aproximadamente 2.000 ha de frijol, 730 ha de maíz, y 300 ha de arroz (Araya y Hernández 2006). Los suelos son ultisoles de baja fertilidad en su mayoría. El área cultivada promedio es $1-5,2$ y 0,2 ha de frijol, maíz y arroz, respectivamente. El sistema de siembra del frijol es a espeque, con densidades de 150.000-160.000 plantas/ha. Sobresale el uso de diversas variedades de frijol simultáneamente, tanto variedades rojas como negras, aunque la mayoría de las áreas sembradas están dedicadas a la producción de cultivares de grano rojo.

El clima se clasifica como tropical lluvioso, e incluye las zonas de vida del bosque muy húmedo tropical en terrenos altos, bosque húmedo tropical en los terrenos más bajos, y bosque húmedo tropical de transición a seco al pie de la Fila Costeña. La precipitación promedio anual es 1.700-1.800 mm, con estaciones lluviosa (abril a noviembre) y seca (diciembre a marzo) bien definidas. Se presenta una disminución en la precipitación a mediados de julio, aprovechada por los productores para realizar la cosecha del frijol. La temperatura promedio es de $22-23^{\circ} \mathrm{C}$, con temperaturas máximas de $28{ }^{\circ} \mathrm{C}$ y mínimas de $18{ }^{\circ} \mathrm{C}$. La frontera agrícola en esta zona ha desplazado los parches boscosos a distancias considerables, lo que ha causado cambios climáticos locales y la carencia de materiales para la construcción (Araya y Hernández 2006).

\section{Diseño}

El ambiente protegido mejorado es consistente con los principios estructurales y funcionales encontrados en los diseños tradicionales, que utilizan los recursos naturales para la solución de sus problemas cotidianos (Figura 1). Las mejoras partieron del examen crítico de las estructuras construidas por los agricultores, conocidas popularmente como "ranchos" (PRIAG 1996), y su mejoramiento a través de la manipulación del balance de energía y la utilización de las propiedades reflectivas de los materiales (Figura 2) (Brown y DeKay 2001). El diseño evaluado posee estética y estructura conceptual moderna (Seale y Wolfe 1981), e incluyó materiales locales de bajo costo. La ventilación cruzada fue mejorada mediante la elevación del piso y la eliminación de las paredes laterales. La adaptabilidad del modelo propuesto a relieves irregulares y la facilidad para su instalación y remoción fueron evaluadas en pruebas preliminares realizadas en la Estación Experimental Fabio Baudrit en Alajuela.

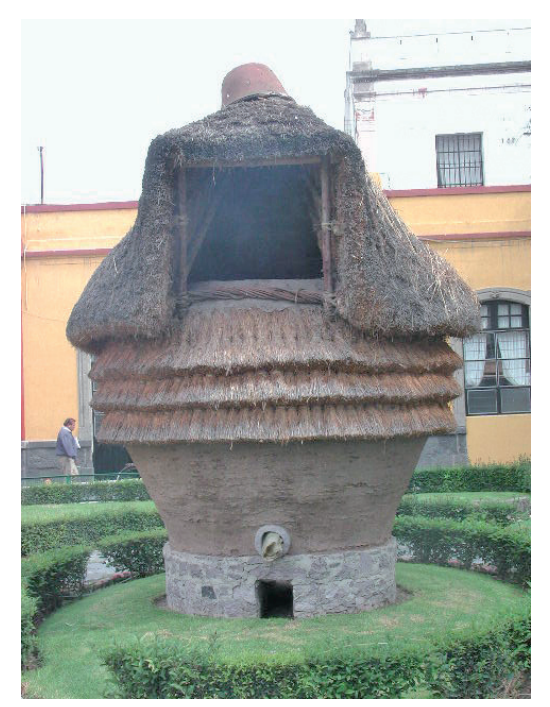

Figura 1. Principios estructurales y funcionales involucrados en el diseño de ambientes protegidos construidos por los aborígenes mesoamericanos, que incluyen alta diversidad arquitectónica, la manipulación del balance de energía, el levantamiento de las estructuras sobre el suelo, la ventilación cruzada, y el uso de materiales aislantes biodegradables, localmente disponibles como barro y zacate. Jardines de la Universidad de Chapingo, México. 2004.

En el campo, se cosecharon plantas de frijol de la variedad Cabécar de color rojo claro (Rosas et al. 


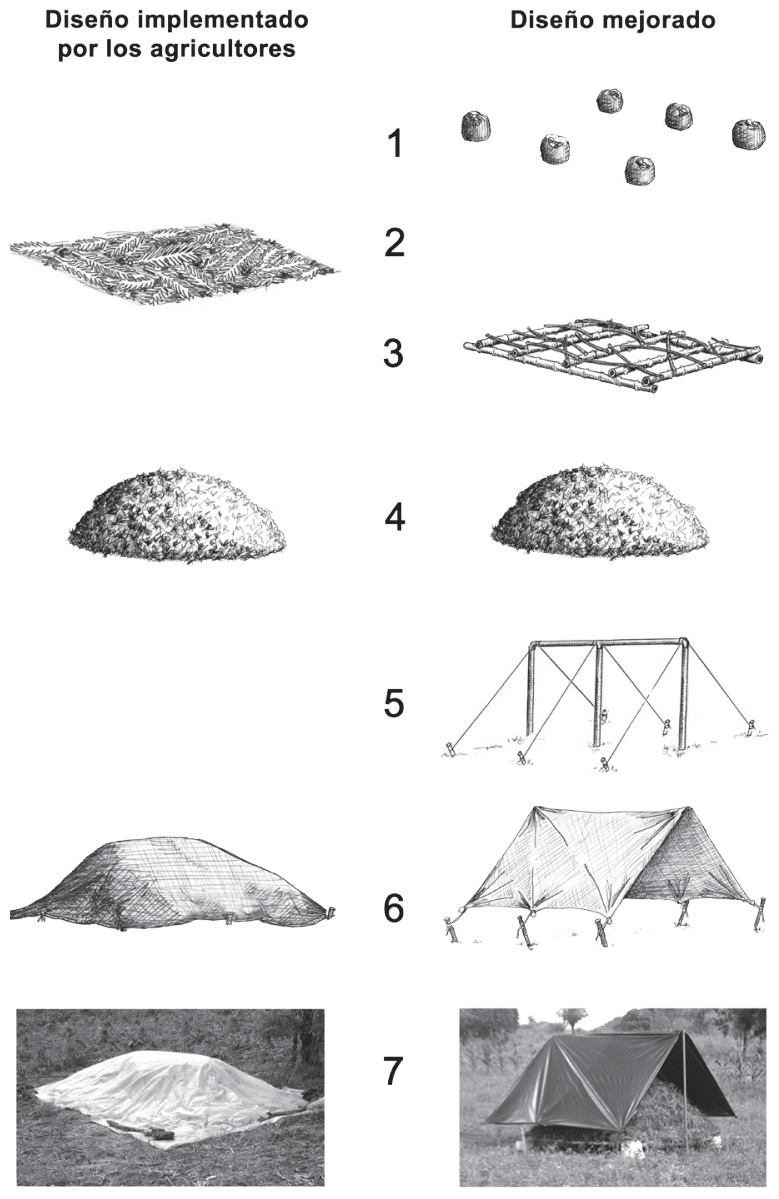

Figura 2. Diseño mejorado obtenido mediante métodos participativos implementados en talleres y en las fincas de los agricultores. 1, 2 y 3) soportes para el levantamiento de la estructura por encima del suelo $(0,25-0,50 \mathrm{~m})$ compuestos por materiales livianos como tubos plásticos o bambú, y un piso de $6 \times 7 \mathrm{~m}$ constituido por materiales vegetales como rastrojos secos u hojas de palmas. 4) montículos ("motetes") formados por plantas de frijol recién cosechadas. 5) estructura de sostén para la cobertura de los montículos, compuesta de tubos plásticos o bambú, de aproximadamente $2,5 \mathrm{~m}$ de altura. 6) coberturas de plástico transparente o materiales reflectivos, respectivamente. 7) "ranchos" instalados en el campo por los agricultores, en contraposición con el diseño bioclimático mejorado, respectivmente. Pérez Zeledón y Buenos Aires, Puntarenas, Costa Rica. 2007.
2004). Las plantas cosechadas permanecieron en el campo por dos a tres días para reducir su contenido de humedad, y luego se depositaron en forma de montículos ("motetes") de aproximadamente 6 x 7 m y 2,25 $\mathrm{m}$ altura, dentro de los ambientes protegidos (Figura 2). Los montículos o motetes se cubrieron con plástico transparente, negro o gris, de $0,0038 \mathrm{~cm}$ (1,50 milésimas de pulgada) de grosor, y se compararon con los "ranchos" construidos por los agricultores, que solo utilizan plástico transparente.

\section{Mediciones ambientales}

Se emplearon HoBos (Onset, modelo H08-00402) programados para registrar la temperatura y la humedad relativa del aire en el exterior y en el interior, y almacenar promedios de intervalos de 10 minutos, en dos localizaciones dentro de los motetes de frijoles. Las mediciones superficiales y profundas fueron realizadas a 0,20 y 1,25 m dentro de los motetes, respectivamente, durante un total de 16 días, resultado de dos períodos de mediciones de ocho días consecutivos cada uno, obtenidos en dos fincas.

\section{Humedad y tiempo de cocción de los granos}

Las mediciones del contenido de humedad de los granos se realizaron en el campo con un determinador de humedad de granos (Seedburo 1200 Series), y el tiempo de cocción se determinó en el Laboratorio de Calidad Agrícola del Consejo Nacional de Producción de Costa Rica, según Decreto No. 32149 MEIC-SMAG, publicado en la Gaceta No. 3 del miércoles 5 de enero del 2005, análisis efectuado a una altitud de 1.000 a 1.250 msnm.

\section{Estadística}

Se realizaron comparaciones de las medias de la temperatura, la humedad relativa, y la calidad de los granos entre los tratamientos. Se aplicaron análisis de varianza y pruebas de significancia utilizando la prueba de Tuckey para separar las medias (Statistica 6.0, StatSoft Inc., 2001). Se utilizó MS Excel para producir las regresiones entre la temperatura, el contenido de humedad y el tiempo de cocción de los granos. 


\section{RESULTADOS Y DISCUSIÓN}

Las mediciones mostraron que se generan pronunciados gradientes de temperatura en el interior de los montículos, con una clara diferenciación entre la superficie y el interior de los mismos, particularmente alrededor del medio día. La temperatura durante el transcurso del día fue superior en las capas superficiales de los montículos, que experimentaron temperaturas máximas de más de $50{ }^{\circ} \mathrm{C}$, y las de mayor profundidad, que alcanzaron temperaturas máximas menores a $\operatorname{los} 30^{\circ} \mathrm{C}$ (Figura 3). Las mayores temperaturas se registraron dentro de las estructuras implementadas por los agricultores y en los ambientes protegidos construidos con plástico transparente, que superaron la temperatura exterior. En contraste, los diseños mejorados construidos con plásticos negro y gris experimentaron temperaturas más moderadas $(29,15$ y $30,55^{\circ} \mathrm{C}$, respectivamente). Los valores promedio de temperatura máxima, mínima y media fueron significativamente superiores ( $\leq \leq 0,05 \%)$ en el interior de los ambientes protegidos construidos por los agricultores, con cobertura transparente, y en el exterior, que en los ambientes protegidos construidos con plástico negro y gris (Figura 4).

La humedad relativa se mantuvo cerca del 100 $\%$ durante la noche en todos los tratamientos, pero permaneció alta (mayor al $90 \%$ ) durante el día sólo en los ambientes protegidos construidos con plástico negro y gris (Figura 3). Las estructuras construidas por los agricultores y los ambientes protegidos con plástico transparente experimentaron reducciones marcadas de la humedad del aire durante el día, que probablemente contribuyeron a la rápida deshidratación del material vegetal almacenado. La humedad relativa decreció a lo largo del día conforme la temperatura del aire aumentó,
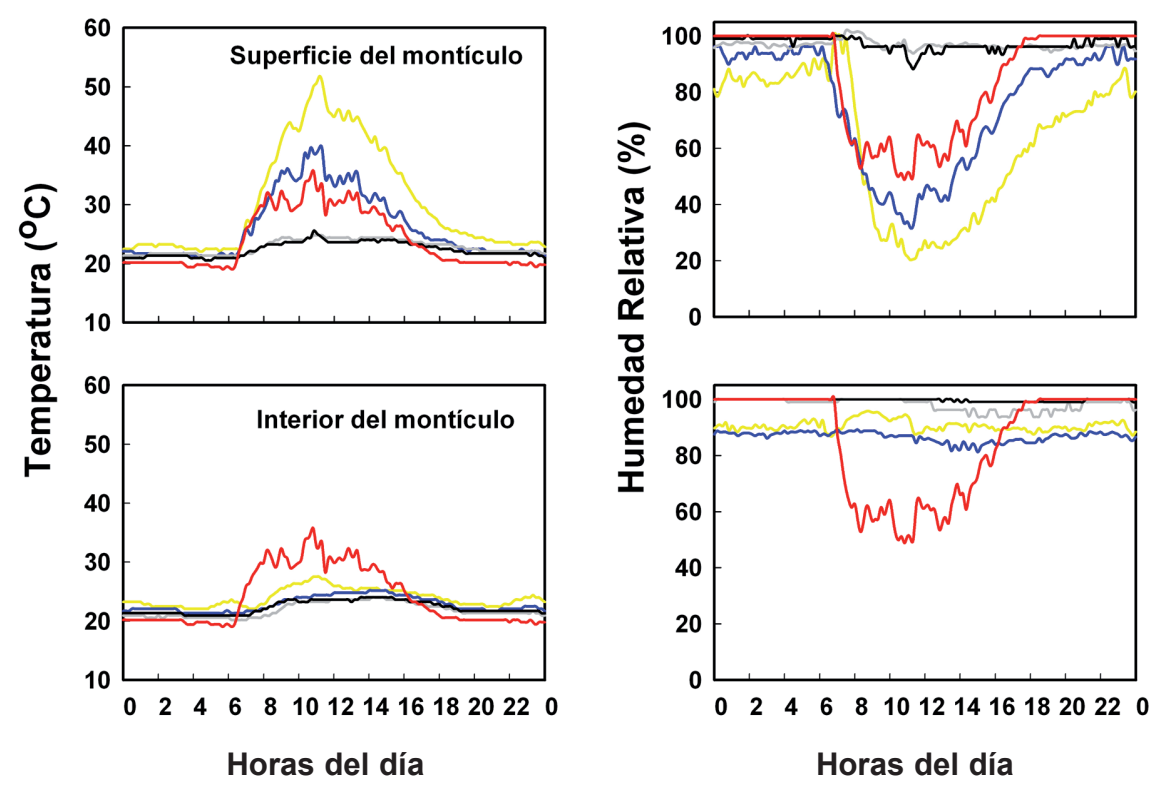

Figura 3. Condiciones de temperatura y humedad relativa medidas durante un día representativo en el interior de ambientes protegidos bioclimáticos construidos con coberturas plásticas de diferentes colores, en relación con la construcción del agricultor y el ambiente externo, y en dos posiciones en el interior de los montículos de material vegetal almacenado. Las mediciones superficiales y profundas se realizaron a 0,20 y $1,25 \mathrm{~m}$ dentro de los montículos, respectivamente. Los trazos rojo, amarillo, azul, negro y gris corresponden al exterior, los "ranchos" de los agricultores, y los ambientes bioclimáticos construidos con plástico transparente, negro y gris, respectivamente. Pérez Zeledón y Buenos Aires, Puntarenas, Costa Rica. 2007. 


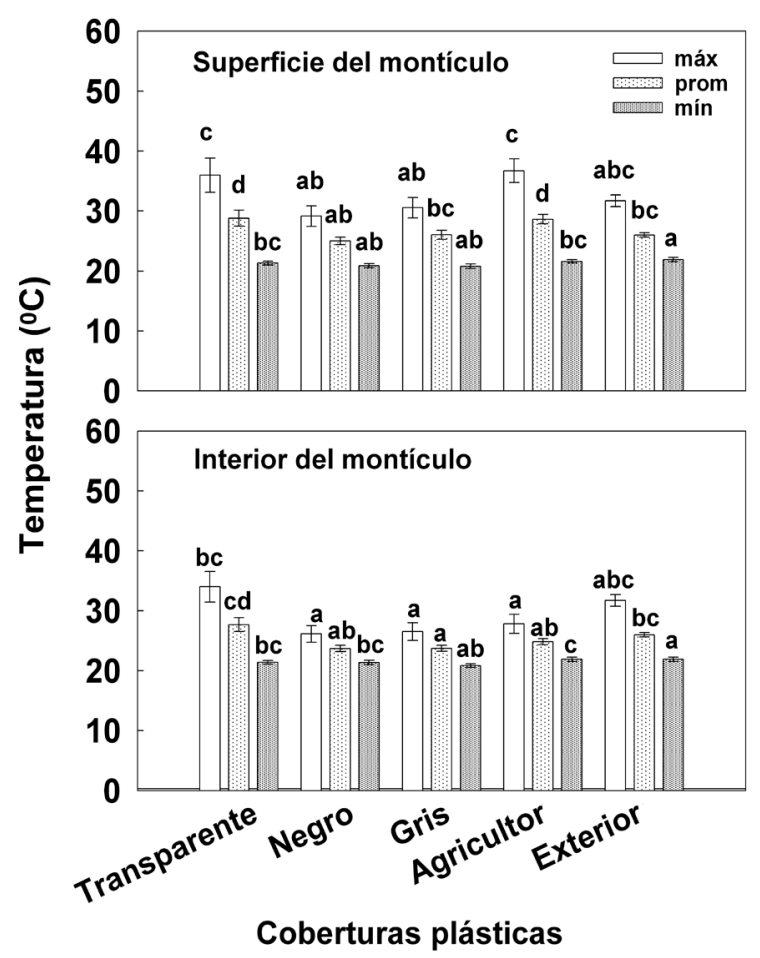

Figura 4. Temperatura máxima, promedio y mínima en el interior de los montículos de frijoles almacenados en ambientes protegidos con coberturas plásticas de diferentes colores, en relación con el ambiente externo, y en dos posiciones en el interior de los montículos. Las mediciones superficiales y profundas se realizaron a 0,20 y $1,25 \mathrm{~m}$ dentro de los montículos de frijoles, respectivamente. Cada valor es el promedio \pm ES de 16 días de observaciones. Letras diferentes indican diferencias significativas entre los tratamientos. Pérez Zeledón y Buenos Aires, Puntarenas, Costa Rica. 2007.

por lo que la pérdida de humedad de los granos fue mayor en los ambientes protegidos que experimentaron las mayores temperaturas. Estas reducciones en la humedad del aire fueron mucho más marcadas en la superficie donde la humedad decreció hasta $30 \%$, que en el interior de los motetes donde la humedad alcanzó el $50 \%$, y fue más pronunciada en las construcciones de los agricultores (Figura 3). La humedad relativa máxima no varió significativamente $(\mathrm{p} \leq 0,05 \%)$ entre los tratamientos y diseños examinados (Figura 5), pero los valores promedio y mínimos fueron

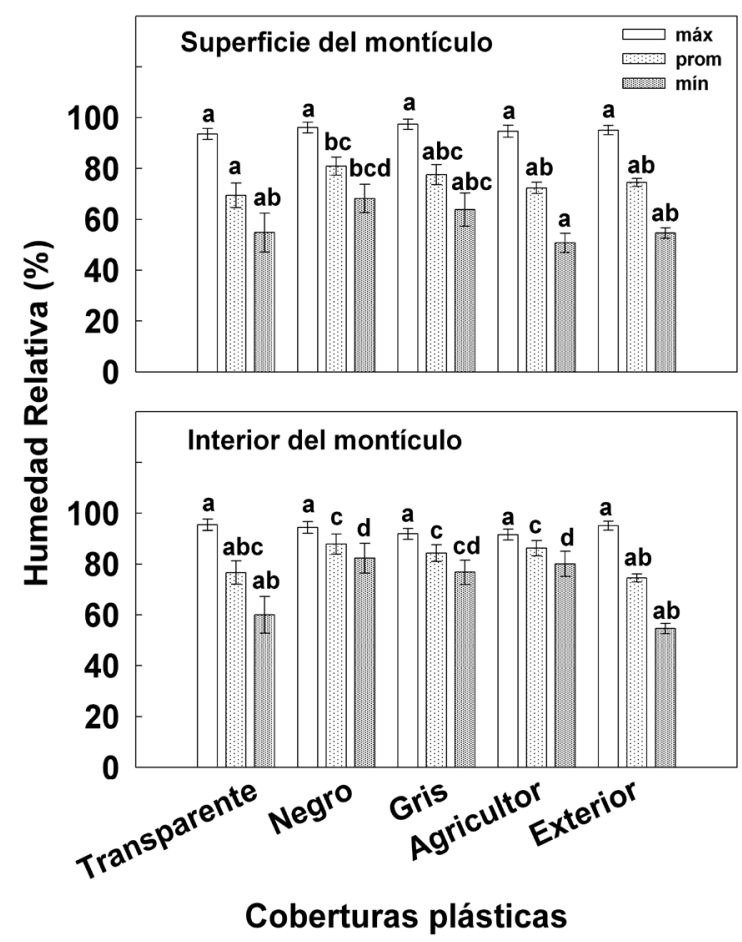

Figura 5. Humedad relativa en el interior de montículos de frijoles almacenados en ambientes protegidos con coberturas plásticas de diferentes colores, en relación con el ambiente externo, y en dos posiciones en el interior del material vegetal almacenado. Las mediciones superficiales y profundas se realizaron a 0,25 y $1,25 \mathrm{~m}$ dentro de los montículos de frijoles, respectivamente. Cada valor es el promedio \pm ES de 16 días de observaciones. Letras diferentes indican diferencias significativas entre los tratamientos. Pérez Zeledón y Buenos Aires, Puntarenas, Costa Rica. 2007.

significativamente menores ( $\mathrm{p} \leq 0,05 \%)$ en el exterior, en el interior de los diseños del agricultor y en las estructuras construidas con plástico transparente, tanto en la superficie como en el interior de los montículos. Los ambientes protegidos construidos con plástico negro y gris mostraron menores reducciones de la humedad relativa durante el día, debido al menor calentamiento experimentado en el interior de los mismos.

Se observó una relación inversa entre el contenido de humedad de las granos y el tiempo de cocción (Figura 6A). Esta relación fue mediada por las altas 

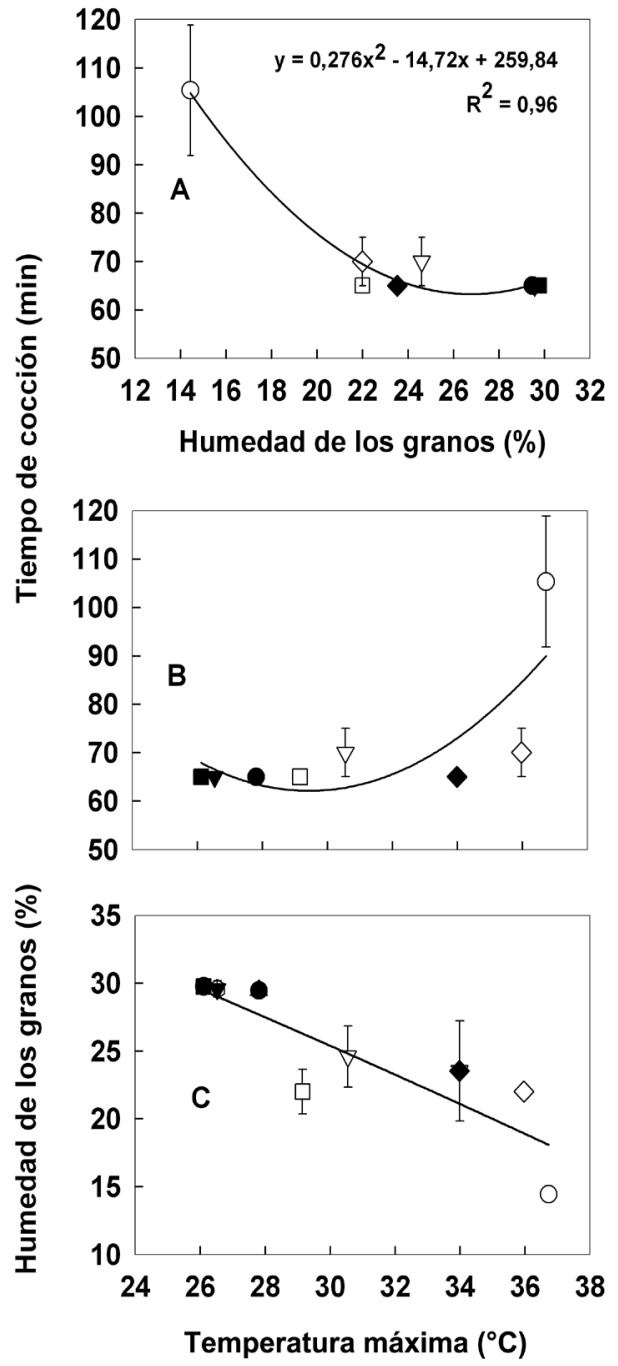

Figura 6. Efecto de la temperatura de los montículos de frijoles sobre el contenido de humedad y el tiempo de cocción de los granos almacenados en ambientes protegidos de diferentes colores durante ocho días en el campo. Cada valor es el promedio \pm ES de 16 observaciones. Los símbolos representan los tratamientos: Oagricultor, superficie; $\bullet$ agricultor, interior; $\diamond$ transparente, superficie; $\diamond$ transparente, interior; $\square$ negro, interior; $\square$ negro, superficie; $\nabla$ gris, interior; $\nabla$ gris, superficie. 2007. temperaturas experimentadas por los frijoles durante el almacenamiento (Figura 6B) y las reducciones rápidas y sustanciales en el contenido de humedad de los mismos (Figura 6C).

Estos resultados indican que períodos cortos de exposición a alta temperatura y reducida humedad pueden resultar en el endurecimiento de los granos y en incrementos del tiempo de cocción. La información disponible indica que en el frijol común y otras leguminosas de grano, el endurecimiento es un fenómeno complejo que implica la cubierta seminal, las paredes celulares y los carbohidratos contenidos en diferentes partes de las semillas (de León et al. 1989, Reyes-Moreno y Paredes-López 1993, Reyes-Moreno et al. 2000, Shiga et al. 2004, Stanley et al. 2007), y es evidente la necesidad de diseñar prácticas que contribuyan a la solución de este problema, en diferentes eslabones de la cadena de producción y distribución del frijol.

Las modificaciones introducidas en el diseño empleado por los agricultores mejoraron el funcionamiento de los ambientes protegidos, aunque mejorías adicionales pueden obtenerse a través de la selección de los materiales y los colores de las cubiertas que componen el techo. Investigaciones futuras deben determinar: a) el tiempo máximo de permanencia de los granos en el campo antes de que se detecten aumentos significativos en el tiempo de cocción, b) la manipulación del balance de energía con otros materiales reflectivos utilizados en las coberturas, biodegradables y de mayor duración, c) el grosor de la capa superficial de los montículos o "motetes", donde el endurecimiento de los frijoles es más severo, y d) la efectividad del uso de materiales vegetales inertes como coberturas sobre los montículos, para reducir el grosor de la capa superficial o eliminarla completamente. La instalación de dos o tres protectores bioclimáticos por hectárea, dependiendo de la cosecha, es necesaria para proveer protección a los granos cosechados y preservar su calidad durante el almacenamiento temporal en el campo.

La modalidad de diseño participativo implementada pone de manifiesto la importancia de recuperar las técnicas tradicionales de la extensión agrícola basadas en el conocimiento del contexto sociológico de las 
comunidades rurales (Araya y Hernández 2006). Es también necesario promover el conocimiento de los principios básicos de la ingeniería rural (van Lengen 2004) y aplicarlos en el diseño de estructuras agrícolas bioclimáticas y sostenibles como establos, porquerizas, gallineros y silos, entre otros.

\section{AGRADECIMIENTOS}

Se agradece a los agricultores Orlando García, Francisco Vega y Marvin Mora por facilitar el trabajo en sus fincas, a Julio Vega y José Miguel Araya por su contribución al diseño e instalación en el campo, a Adrián López por su ayuda en la realización del ensayo, a Álvaro Chaves y María Barrantes de la Dirección de Calidad Agrícola del Consejo Nacional de Producción, por los datos del tiempo de cocción, a Julio Arias por su colaboración en el análisis estadístico, y al Proyecto de Granos Básicos UPIAV - Reconversión Productiva por el financiamiento parcial de este estudio.

\section{LITERATURA CITADA}

Araya, R; Hernández, JC. 2006. Mejora genética participativa de la variedad criolla de frijol "Sacapobres". Agronomía Mesoamericana 17(3):347-355.

Bertsch, F; Monreal, C. (eds). 1996. El uso sostenible del suelo en zonas de ladera: el papel esencial de los sistemas de labranza conservacionista. Red Latinoamericana de Labranza Conservacionista (RELACO). San José, Costa Rica. 309 p.

Bewley,JD; Black, M. 1994. Seeds; physiology of development and germination. New York, Plenum Press. 445 p.

Brown, GZ; DeKay; M. 2001. Sun, wind and light; architectural design strategies. New York, John Willey. 381 p.

Centro Internacional de Agricultura Tropical (CIAT). 1980 Semilla de frijol de buena calidad. 2 ed. Cali, Colombia. Centro Internacional de Agricultura Tropical (CIAT). Serie 04.SB-12.03. $37 \mathrm{p}$.

de León, LF; Brezan R; Elías, LG. 1989. Effects of the seed coat on the hardening of common beans (Phaseolus vulgaris). Arch. Latinoam. Nutr. 39(3):405-418.

Gwinner J; Harnisch, R; Mück, O. 1996. Manual of prevention of post-harvest grain losses. Post-harvest project. GTZ, Eschborn, Germany. 388 p.
Mora, M. 1981. Pequeña secadora de leña para granos. Agronomía Costarricense 5(1/2):131-133.

Mora, M. 1982. Influencia de diferentes temperaturas y contenidos de humedad sobre el tiempo de cocción del frijol (Phaseolus vulgaris L.) almacenado durante 18 meses. Agronomía Costarricense 6(1/2):87-89.

Mora, M. 1989. Comparación del tiempo de cocción de ocho cultivares de frijol común (Phaseolus vulgaris L.). Boltec (Costa Rica) 22(2):32-36.

Pastor-Corrales, M; Schwartz, HF. 1994. Problemas de producción del frijol en los trópicos. Cali, Colombia. Centro Internacional de Agricultura Tropical (CIAT). Serie 09SB-1. 734 p.

Programa Regional de Reforzamiento de la Investigación Agronómica (PRIAG). 1996. Después de la cosecha maneje correctamente el frijol. Manejo Poscosecha. Ministerio de Agricultura y Ganadería de Costa Rica. 8 p.

Reyes-Moreno, C; Paredes-López, O. 1993. Hard-to-cook phenomenon in common beans - a review. Crit. Rev. Food Sci. Nutr. 33(3):227-286.

Reyes-Moreno, C; Milán-Carrillo, J; Amienta-Rodelo, E; Okamura-Esparza, J. 2000. Influence of storage at high temperature and high humidity on seed quality of chickpeas (Cicer arietinum L.). Food Sci. and Technol. Intern. 6(6):473-482.

Rosas, JC; Beaver, JS; Escoto, D; Pérez, CA; Llano, A; Hernández, JC; Araya, R. 2004. Registration of “Amadeus 77”, small red common bean. Crop Sci. 44:1867-1868.

Sánchez-Trejos, P. 2006. Comentarios sobre métodos para medir la dureza del frijol. Memorias del IX Congreso del Sector Frijolero. Programa de Investigación y Transferencia en Tecnología Agropecuaria - Frijol (PITTA - Frijol). Alajuela, Costa Rica. p. 59-66.

Seale J; Wolfe, J. 1981. Modeling and design of future bioshelters. J. New Alchemist 7:112-118.

Shiga T; Lajolo FM; Tullia MC; Filisetti C. 2004. Changes in cell wall polysaccharides during storage and hardening of beans. Food Chemistry 84(1):53-64.

Stagno, B; Gutiérrez-Soto, MV. 2005. Harnessing comfort through climate. Performance of the Holcim building. A case study in Costa Rica. The 2005 World Sustainable Building Conference, Tokyo. p. 1545-1552.

Stanley, DW; Michaels, TE; Plhak, LC; Caldwell, KB. 2007. Storage-induced hardening in 20 common bean cultivars. J Food Qual 13(4):233-247.

van Lengen, J. 2004. Manual do arquiteto descalço. Rio de Janeiro, Livraria do Arquiteto. 697 p. 